\title{
Hemorrhagic shock secondary to portal hypertensive duodenopathy complicated by lupus and autoimmune liver cirrhosis
}

\author{
Samer Alkhuja, Jonathan Goldner \\ Department of Medicine, The Commonwealth Medical College, Pocono Medical Center, East Stroudsburg \\ Email address: \\ alkhuja@yahoo.com(S. Alkhuja),jgoldner@pmchealthsystem.org(J. Goldner)
}

\section{To cite this article:}

Samer Alkhuja, Jonathan Goldner. Hemorrhagic Shock Secondary to Portal Hypertensive Duodenopathy Complicated By Lupus and Autoimmune Liver Cirrhosis. American Journal of Internal Medicine. Vol. 1, No. 3, 2013, pp. 25-26. doi: 10.11648/j.ajim.20130103.11

\begin{abstract}
Systemic lupus erythematosus is a common autoimmune disorder that frequently is complicated by additional autoimmune diseases. There is a correspondence between hepatic diseases and systemic lupus erythematosus that ranges from subclinical elevations of liver enzymes to hepatic infarction. We present a rare case of portal hypertensive duodenopathy in a patient with autoimmune hepatitis/liver cirrhosis associated with systemic lupus erythematosus. Patients with systemic lupus erythematosus who present with upper gastrointestinal bleed should be evaluated for abnormal liver functions and have portal hypertensive duodenopathy included in the differential diagnosis.
\end{abstract}

Keywords: Shock, Lupus, Liver Cirrhosis, GI Bleed

\section{Case Presentation}

A 42 year old women presented to the emergency department (ED) with dizziness, nausea and having melena for one day. She has a past medical history of systemic lupus erythematosus (SLE), gastric esophageal reflux disease, cholelithiasis, chronic anemia, asthma, biopsy proven autoimmune hepatitis (AIH), and liver cirrhosis. Her past surgical history includes an esophagogastroduodenoscopy (EGD), which was normal, and a liver biopsy two years ago. Test for antinuclear antibodies was positive.

Her home medications include prednisone, azathipioprine, lansoprazole, hydroxycloroquine and albuterol. The patient is a smoker of 6-7 cigarettes/day. She denied alcohol consumption or allergies. Her family history was significant for diabetes mellitus, hypertension, and hypercholestremia. Physical examination, in the ED, revealed blood pressure (BP) $77 / 49 \mathrm{mmHg}$, pulse $132 /$ minute, respiration $22 /$ minutes, temperature $37.5^{0}$ Celsius. The reminder of her examination was unremarkable. Blood pressure improved with intravenous (IV) fluids and blood transfusion, with subsequent systolic BP measurements in the $90 \mathrm{~s} \mathrm{mmHg}$.

Laboratory findings were: Complete blood count showed white blood cell 8,000 (normal range 4200 - 10,200), hemoglobin $7.0 \mathrm{~g} / \mathrm{dL}$ (normal range 12-15), hematocrit 20.8\% (normal range 37-47\%), platelet 99,000 (normal range 140,000 - 450,000). Prothrombin time was 19 seconds (normal range 9.0 - 12.0) Activated partial thromboplastin time was 41 seconds (normal range $24-36$ ), and International normalized ration 1.8 (normal range $0.9-1.1$ ). AST 131 IU/L (normal range 15- 41), ALT 81 IU/L (normal range $17-68$ ), total bilirubin $0.9 \mathrm{mg} / \mathrm{dL}$ (normal range 0.3 1.2 ), direct bilirubin $0.3 \mathrm{mg} / \mathrm{dL}$ (normal range $0.1-0.5$ ), alkaline phosphatase $48 \mathrm{IU} / \mathrm{L}$ (normal range 38 - 126), albumin $1.6 \mathrm{~g} / \mathrm{dL}$ (normal range $3.5-5.0$ ), amylase 61 IU/L (normal range $28-100$ ), lipase $20 \mathrm{IU} / \mathrm{L}$ (normal range $22-51$ ), and alpha-fetoprotein $1.8 \mathrm{ng} / \mathrm{ml}$ (normal range $0.5-9.0)$.

Electrocardiogram showed sinus tachycardia. Computed tomography (CT) scan of abdomen and pelvis showed the spleen measuring $9.3 \mathrm{~cm}$, gallbladder demonstrates calculi, liver showed nodular contour suggesting cirrhosis. Chest radiograph showed blunting of the right costophrenic angle.

While in the intensive care unit, despite treatment with IV fluid and blood transfusions, the patient's BP measurements were in the $70 \mathrm{~s} \mathrm{mmHg}$. Intubation was performed for airway protection in view of hemodynamic instability secondary to hemorrhagic shock, and lethargy which was followed by unresponsiveness. An EGD was performed and demonstrated normal findings on inspection of esophagus and stomach. Examination of second and 
third portion of the duodenum showed an actively bleeding duodenal varix (DV). Lavage was performed and injection of $6 \mathrm{~mL}$ of ethamolin $5 \%$ into the DV for temporary homeostasis was performed. The patient was kept intubated until the achievement of hemodynamic satbiliy, and immediately underwent placement of transjugular intrahepatic portosystemic shunts which resulted in permanent homeostasis and resolving of hemorrhagic shock, and shortly after was extubated. The patient was discharged home six days after presentation.

\section{Discussion}

SLE is a common autoimmune disorder characterized by multisystem involvements and complicated by other autoimmune disease. Hepatic involvement in patients with SLE has been reported. Elevated levels of transaminases associated with SLE was reported in $1 / 3$ of patients reportedly by Miller et al. (1), and in 19 (23.5\%) of 81 patients reported by Gibson et al. (2).

In a review of 40 patients with liver enzymes abnormalities and SLE, 6 were found to have AIH and 3 had cirrhosis (3). Runyon et al. reported only four (1.7\%) of 238 patients with SLE to have AIH or cirrhosis (4). A review by Mastsumoto et al. autopsy registry data for 1,468 patients with SLE indicates that the incidence of chronic liver disease in SLE autopsy is AIH, 2.4\%; cirrhosis, 1.1\%; and liver fibrosis, $0.8 \%$ (5). AIH complicated by SLE should be considered not as a part of the range of AIH, but rather as a category of AIH-SLE overlap (6).

Gastrointestinal (GI) manifestations of SLE were reviewed by $\mathrm{Xu}$ et al., and were found in 39 (22\%) of 177 consecutive SLE patients (7). GI complications occurred as the initial symptoms in 12 patients (12/39). Four patients $(10.3 \%)$ in the group with GI complications died. While the mortality rate in SLE without GI complications was only $2.2 \%$, this suggests that SLE patients with GI manifestations have worse prognosis than those without (7).

Portal hypertensive duodenopathy (PHD) is rare and was found only in $46(8.4 \%)$ of 549 patients with cirrhosis and portal hypertension who underwent EGD (8). Mastusi et al. documented in a review of 12 patients with endoscopically DVs their underlying disease consisted of liver cirrhosis in 8 patients, and pancreatic cancer-related pylemphraxis in 4 patients (8). DVs are uncommon and account only for $5 \%$ of variceal hemorrhages (9), they often have a fatal outcome with a mortality as high as $40 \%$ (9). Verbeeck et al. reported successful temporizing treatment by precutaneous transhepatic embolization (9). Okahara et al. reported successful treatment of DVs by retrograde transvenous obliteration with additional or alternative techniques as a result of the complex hemodynamic features of the DVs (10).

\section{Conclusion}

PHD although rare but it may present as hemorrhagic shock as a result of upper GI bleed secondary to DV in a patient with AIH/liver cirrhosis. PHD should be considered in the differential diagnosis of an SLE patient with liver cirrhosis who is presenting with an upper GI bleed.

\section{References}

[1] M.H. Miller, M.B. Urowitz, D.D Gladman, L.M Blendis, "The liver in systemic lupus erythematosus", Q. J. Med., Summer 1984,Vol.53(211),pp.401-409.

[2] T. Gibson, A.R. Myers, "Subclinical liver disease in systemic lupus erythematosus", J. Rheumatol., Sep-Oct 1981,Vol.8(5),pp.752-759.

[3] V.R. Chowdhary, C.S. Crowson, J.J. Poterucha, K.A. Moder,'Liver involvement in systemic lupus erythematosus: care review of 40 patients", J. Rheumatol., Nov 2008,Vol.35(11),pp.2159-2164.

[4] B.A. Runyon, D.R. LaBrecque, S. Anuras, "The spectrum of liver disease in systemic lupus erythematosus. Report of 33 histologically proved cases and review of the literature", Am. J. Med., Aug 1980,Vol.69(2),pp.187-194.

[5] T. Matsumoto, T. Yoshimine, K. Shimouchi, H. Shiotu, N. Kuwabara, Y. Fukuda, T. Hoshi,'The liver in systemic lupus erythematosus: pathologic analysis of 52 cases and review of Japanese autopsy registry data", Hum Pathol., Oct 1992,Vol.23(10),pp.1151-1158.

[6] J. Tojo, H. Ohira, K. Abe, J. Yokokawa, J. Takiguchi, T. Rai, S. Shishido, Y. Sato, R. Kasukawa, " Autoimmune hepatitis accompanied by systemic lupus erythematosus", Intern. Med., Mar 2004,Vol.43(3),pp.258-262.

[7] D. Xu, H. Yang, C-C. Lai, P. Li, X. Zhang, X-O. Yang, F-C. Zhang, J-M. Qian,"Clinical analysis of systemic lupus erythematosus with gastrointestinal manifestations", Lupus., June 2010,Vol.19(7),pp.866-869.

[8] S. Matsui, M. Kudo, T. Ichikawa, M. Okada, Y. Miyabe, "The clinical characteristics, endoscopic treatment, and prognosis for patients presenting with duodenal varices", Hepatogastroenterology., May-Jun 2008,Vol.55(84),pp.959962.

[9] N. Verbeeck, P. Stammet, J. Weber, L. Mertens, J. Se'rignol, "Bleeding duodenal varices, an unusual presentation of portal hypertension: 3D MSCT of the feeding branches facilitates temporizing treatment by percutaneous transhepatic embolization", JBR-BTR., Nov-Dec 2009,Vol.92(6),pp.289-292.

[10] M. Okahara, H. Kiyosue, S. Ueda, J. Kashiwagi, S. Tanoue, N. Hongo, H. Mori, "Anatomic features and retrograde transvenous obliteration of duodenal varices associated with mesocaval collateral pathway", J. Vasc. Interv. Radiol., Oct 2012,Vol.23(10),pp.1339-46. 